\title{
To analyze the clinical characteristics of emergency syncope patients and explore their clinical treatment strategies
}

\author{
XIE Chuyu ${ }^{1}$, ZHANG Hujie ${ }^{1}$, HUANG Xuanjie, HUANG Jianmao, MO Zhifeng ${ }^{*}$ \\ The Emergency and Disaster Medical Center, The Seventh Affiliated Hospital, Sun Yat-sen \\ University, Shenzhen, China, 518107 \\ ZHANG Hujie is co-first author. * MO Zhifeng is the corresponding author. \\ Email:mozhf3@mail.sysu.edu.cn.https://orcid.org/0000-0002-9273-003X
}

Article History Received 5 June 2021 Accepted 25 June 2021 Published 30 September 2021 Cite this Article XIE Chuyu, ZHANG Hujie, HUANG Xuanjie, HUANG Jianmao, MO Zhifeng. To analyze the clinical characteristics of emergency syncope patients and explore their clinical treatment strategies [J].Medical Research, 2021.3(3):1-8, http://dx.doi.org/mrhk/10.6913/MRHK.2 02109_3(3).0001

Copyright $(2021$ Creative Publishing Co., Limited.All rights reserved.Email: mrhk@mrhk.cc

\begin{abstract}
Objective To analyze the clinical characteristics of emergency syncope patients and explore the strategies in their clinical treatment.

Methods Eighty-five patients with emergency syncope admitted to our hospital from January 2019 to April 2021 were reviewed. The clinical data of all patients were retrospectively analyzed. The common causes were analyzed and summarized, and the strategies in clinical treatment were proposed.

Results The main causes of syncope are neuroreflex syncope, follow by cardiac syncope, cerebral syncope, orthostatic hypotension syncope, hysterical syncope and unexplained syncope. They accounted for $35.3 \%, 30.6 \%, 15.3 \%, 12.9 \%, 3.5 \%$, and $2.4 \%$, respectively. Conclusion Emergency department physicians should evaluate emergency syncope patients in time, identify patients as soon as possible, confirm the etiological diagnosis, and give reasonable and effective symptomatic treatment and treatment measures in order to improve the prognosis and ensure the life safety and quality of patients.
\end{abstract}

Keywords Emergency; Syncope; Clinical characteristics; Treatment strategy

\section{INTRODUCTION}

Syncope is a transient loss of consciousness and loss of physical control resulting from sudden, transient decreases in whole brain tissue perfusion .Syncope is characterized by rapid onset, short time and spontaneous complete recovery, with typical syncopal episodes generally lasting no more than $20 \mathrm{~s}$. A few lasting several minutes, and a few patients may develop convulsions. ${ }^{[1]}$ In emergency clinical work, acute syncope is a common clinical emergency in the emergency department. Such patients have acute onset, complex and diverse causes, and great differences in the severity of the condition and prognosis. If they cannot be timely and correctly diagnosed and effective emergency treatment methods taken, they 
sometimes delay treatment or even endanger life ${ }^{[2]}$. From January 2019 to April 2021, 85 patients with acute syncope were admitted to our emergency department. The common causes were analyzed and summarized, and the strategies in clinical treatment were proposed. It is reported as follows.

\section{MATERIALS AND METHODS}

\section{General data}

Eighty-five emergency syncope patients were admitted to our hospital from January 2019 to April 2021, including 42 males and 43 females, aged 15-85 years, with an average of $46.7 \pm$ 8.6 years. The patient visited the hospital 10-50 minutes after onset. In this group of patients, 42 patients had a previous history of similar attacks, 43 patients had no history of attacks, 17 patients had a history of heart disease, 8 patients had a history of hypertension, and 5 patients had atrial fibrillation. (Table 1)

\section{Initial Evaluation of Syncope}

Data on the clinical features of transient loss of consciousness (TLOC) are usually obtained from patient as well as eyewitness history taking. Patients who present for the first time with possible TLOC should first have their medical history taken to determine whether they are truly TLOC. TLOC has four specific clinical features: short duration, abnormal motor control, loss of response, and memory loss upon loss of consciousness.

The predominant type of TLOC is generally identifiable by history taking. The following key questions should be answered during the initial assessment: (1) Is this event a TLOC?(3) If it is TLOC, is it syncope or is it non-syncope?If syncope is suspected, is the etiological diagnosis clear? (4) Is there evidence to suggest that there is a high risk of cardiovascular events or death? When seizures or psycho-psychiatric factors are suspected to cause TLOC seizures, appropriate therapeutic measures should be taken. With a detailed history, physicians can differentially diagnose approximately $60 \%$ of patients with syncope from those who develop TLOC. ${ }^{[3]}$ (Figure 1)

\section{Diagnosis of Syncope Based on Initial Assessmen}

A diagnostic assessment for suspected syncope at TLOC is the initial assessment for syncope. It includes (1) Detailed history taking of present and previous attacks, but also in-person or telephone questioning of witnesses; (2) Physical examination, including measurement of blood pressure in supine and standing positions; (3) Electrocardiography. According to the above results, other examination items can be performed when necessary: (1) ECG monitoring should be performed immediately when arrhythmic syncope is suspected; (2) Echocardiography should be performed when there is a previous history of heart disease, suggesting structural heart disease or syncope secondary to cardiovascular causes; (3) Carotid sinus massage examination should be performed in patients aged $>40$ years; (4) Head-up tilt table test should be performed when orthostatic hypotension or reflex syncope is suspected; (5) Blood examination should be performed when there is corresponding clinical evidence.Hematocrit and hemoglobin should be examined when bleeding is suspected.oxygen saturation and blood gas analysis should be examined when hypoxia is suspected.Troponin concentration should be measured when myocardial ischemia-related syncope is suspected, 
and D dimer should be examined when pulmonary embolism is suspected. When the diagnosis of syncope is basically clear without further evaluation, the corresponding treatment plan can be developed. ${ }^{[4]}$

\section{Diagnostic methods}

Syncope has its common characteristics, including the vast majority of sudden onset, after the onset of dizziness, palpitation, nausea and vomiting, pallor, confusion, duration of seconds to minutes will be naturally awake, generalized fatigue and weakness, automatic recoveryfollowed by later, generally no convulsions and urinary incontinence. But a small number of patients may have convulsions; sometimes accompanied by trauma. After emergency syncope patients visit the hospital, ask the patients about their families or escorts of their medical history in detail, including age, causes, inducement, attack frequency, signs, duration, symptoms, clinical manifestations after illness and medication; comprehensive neurological examination, cardiovascular system examination, observe the patient's fundus, upper and lower limb tendon reflexes, body surface skin sensation, eye-closure balance,heart and lung examination, etc. All patients met the diagnostic criteria for syncope ${ }^{[5]}$ : (1)Sudden transient complete loss of consciousness, with a certain self-limitation; (2) Patients can completely recover spontaneously; (3)Rule out epilepsy, hypoglycemia and other related diseases similar to syncope in clinical practice. The diagnosis of syncope was confirmed in all patients without severe cardiac, hepatic, or renal insufficiency.

\section{Statistical methods}

The experimental data were analyzed using the statistical software SPSS 22.0, and the measurement data were expressed as mean \pm standard deviation $(x \pm s)$, using the $t$-test; the enumeration data were expressed as rate, using the $\chi^{2}$ test, and $\mathrm{P}<0.05$ was considered statistically significant.

Table 1 Basic data of patients with syncope

\begin{tabular}{cccccc}
\hline Gender & $\begin{array}{c}\text { Number } \\
(\mathrm{n})\end{array}$ & Hypertension & Diabetes & $\begin{array}{c}\text { Coronary } \\
\text { heart disease }\end{array}$ & $\begin{array}{c}\text { atrial } \\
\text { fibrillation }\end{array}$ \\
\hline male & 42 & 5 & 7 & 11 & 2 \\
female & 43 & 3 & 5 & 6 & 3 \\
\hline sum & 85 & 8 & 12 & 17 & 5 \\
\hline$\chi^{2}$ & & 0.645 & 0.198 & 0.443 & 0.385 \\
P & & 0.445 & 0.662 & 0.556 & 0.478 \\
\hline
\end{tabular}

Figure 1 Flow Chart for Initial Assessment and Risk Stratification of Patients with Syncope

\section{Clinical treatment strategy}

The general principle of formulating the treatment plan according to the risk stratification and specific pathogenesis: the main factor determining the efficacy is the mechanism of syncope; the criteria for determining the efficacy is to observe whether the symptoms recur after treatment; 85 patients with syncope in this group were placed in the supine position, the head was slightly lowered, the buckle was released to maintain airway patency, nasal oxygen inhalation was given, intravenous infusion channel was rapidly established, and the changes 
in vital signs were closely observed. While treating, ask the patient, family member or escort again for missed medical history, predisposing causes, medication before and after illness, and take corresponding therapeutic measures for different causes of syncope. The patient who definitely diagnosed with cardiac syncope, cerebral syncope and unexplained syncope were admitted for further diagnosis and treatment. The remaining patients were discharged with significantly improved symptoms after treatment in the emergency observation room and followed up in the outpatient department.

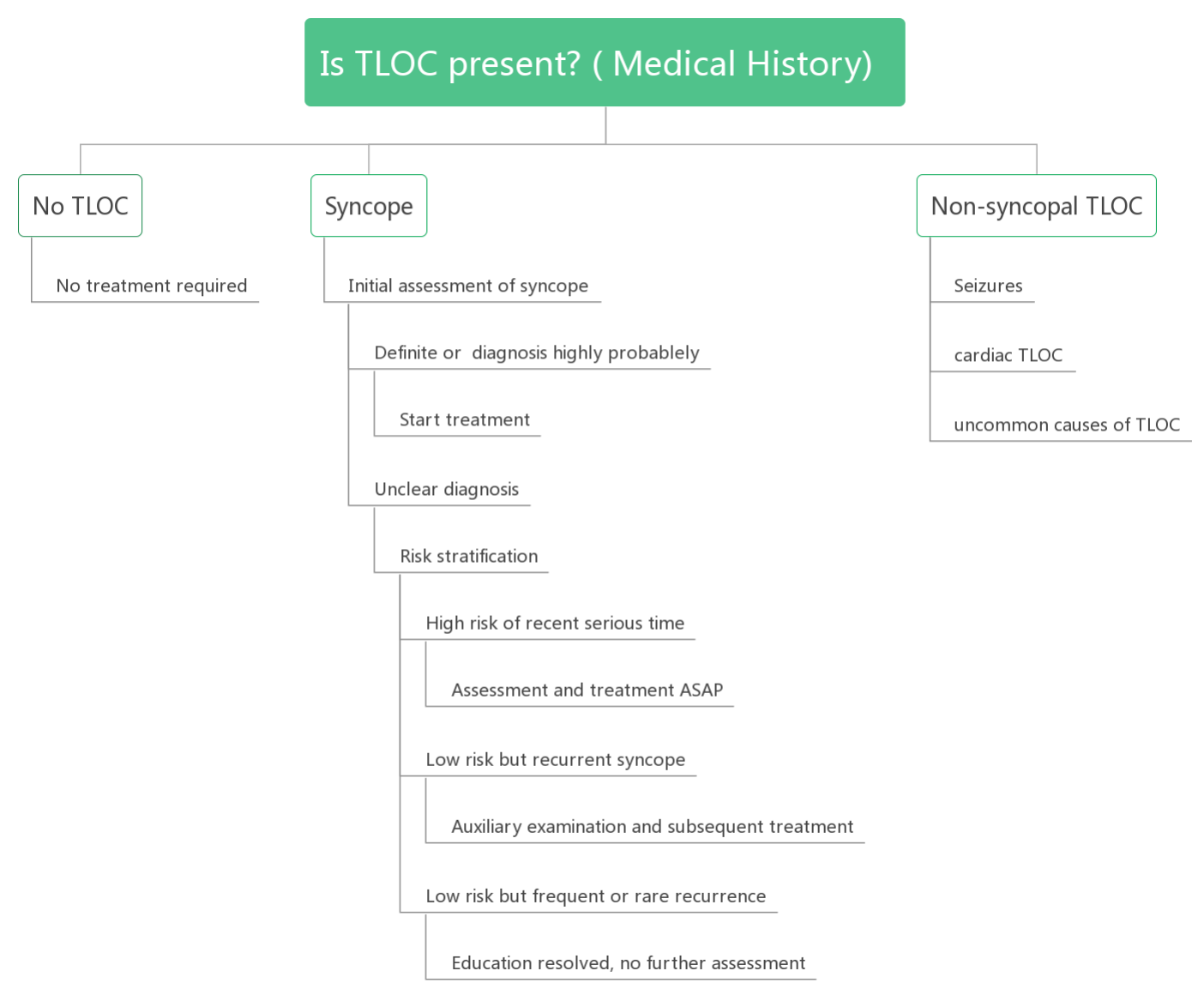

\section{RESULT}

In this group, 85 patients with emergency syncope were diagnosed and treated in the emergency department and admitted to the inpatient department for diagnosis and treatment. Neuroreflex syncope was the the main causes of syncope , which accounted for $35.3 \%$, followed by cardiac syncope(30.6\%), cerebral syncope(15.3\%),orthostatic hypotension syncope (12.9\%), hysterical syncope(3.5\%) and unexplained syncope(2.4\%). Patients definitely diagnosed as cardiac syncope, cerebral syncope, and unexplained syncope were admitted inpatient department for further diagnosis and treatment. The remaining cases were significantly improved and discharged after treatment in the emergency observation room and followed up in the outpatient department. Patients in this group recovered well regardless of diagnosis and treatment in emergency department and in inpatient department. (Table 2)

Table 2 Classification and proportion of patients with syncope 


\begin{tabular}{ccccccc}
\hline & $\begin{array}{c}\text { neuroreflex } \\
\text { syncope }\end{array}$ & $\begin{array}{c}\text { cardiac } \\
\text { syncope }\end{array}$ & $\begin{array}{c}\text { cerebral } \\
\text { syncope }\end{array}$ & $\begin{array}{c}\text { orthostatic } \\
\text { hypotension } \\
\text { syncope }\end{array}$ & $\begin{array}{c}\text { hysterical } \\
\text { syncope }\end{array}$ & $\begin{array}{c}\text { unexplained } \\
\text { syncope }\end{array}$ \\
\hline $\begin{array}{c}\text { Number } \\
(\mathrm{n})\end{array}$ & 30 & 26 & 13 & 11 & 3 & 2 \\
$\begin{array}{c}\text { Proportion } \\
(\%)\end{array}$ & 35.3 & 30.6 & 15.3 & 12.9 & 3.5 & 2.4 \\
\hline
\end{tabular}

\section{DISCUSSION}

Clinical manifestations of syncope is a symptom of cerebral hypoxia and ischemia caused by a variety of reasons, which can cause transient postural muscle tension and loss of consciousness in patients. Some syncopes cause sudden death, which poses a serious threat to the basic health and life safety of patients ${ }^{[6]}$. Therefore, analysis of the causes and symptoms of syncope plays an important role in confirming the diagnosis and then reversing the prognosis in a timely manner ${ }^{[7]}$. In emergency clinical work, the diagnosis of syncope should be rapidly confirmed. First, it is necessary to recognize the inducement of syncope, the frequency of attacks whether there are signs before onset,the clinical manifestations after onset and the medication before and after onset and comprehensive analysis of comprehensive physical examination, relevant auxiliary examination report with this condition.In the process of emergency clinical diagnosis of syncope, other non-syncopal, transient disturbance of consciousness diseases should first be excluded based on the history and symptoms, as well as the results of auxiliary examination, which include syncopal-like diseases without impairment of consciousness, such as falls, hysteria, etc,follow by dizziness, diaphoresis, chest tightness and palpitations and other symptoms of partial or complete loss of consciousness. As an emergency department physician, when admitting patients with syncope, the diagnostic ideas should be rapid and concise in an effort to confirm the diagnosis as much as possible in the shortest time and make effective treatment decisions in a timely manner. General treatment adopts supine position, with slightly lower head and slightly elevated lower limbs to promote blood reflux in the brain, and nasal oxygen inhalation is given to help patients recover consciousness as soon as possible. Those who have confirmed the etiological diagnosis are given corresponding individualized treatment decisions. Patients with syncope that cannot be identified in a short period of time should be admitted for further observation and diagnosis. The diagnosis and treatment of 85 patients with emergency syncope in this group were reviewed, and it was considered that by asking medical history and physical examination, combined with auxiliary examination. Syncope was considered first; the causes of syncopal attacks were considered second. It is important to assess the risk degree of the patient's condition in the diagnosis . Timely clinical risk stratification and clinical assessment and early identification are helpful for the diagnosis and treatment of syncope. ${ }^{[8]}$ If the patient is in critical condition during the first diagnosis, the treatment measures must follow the principles of saving life first, protecting organs second, and restoring function third.Saving life first and then treating disease, treating its symptoms urgently, and treating its root cause slowly that can avoid adverse consequences to the greatest extent. ${ }^{[9]}$

The results of this study showed that there were 30 cases of neuroreflex syncope in 85 
emergency syncope patients, accounting for the highest proportion. In the sultry weather, rapid changes in body position and standing environment that can cause patients with reduced cardiac output and even insufficient blood supply and other conditions, which causes syncope symptoms. Fatigue, hunger, cough and pain are common predisposing factors for this type of syncope patients. Such patients have early symptoms such as headache, nausea, tinnitus, and pale complexion and require prompt medical attention. ${ }^{[10]}$ Environmental and psychological factors should be considered comprehensively in the clinical treatment of patients with neuroreflex syncope, and their pregnancy history and menstrual history should be carefully asked for patients of reproductive age. ${ }^{[11]}$ No special medication is required except to help them regain consciousness as soon as possible. In this study, there were 26 patients with cardiac syncope who ranked second, and the elderly belonged to the high incidence group of this syncope type. Among the many causes of syncope, cardiac syncope is the most serious, and the treatment prognosis is poor, which can cause arrhythmia, myocardial systolic dysfunction and other symptoms. Clinical studies have pointed out that for the treatment of patients with cardiac syncope, attention should be paid to the analysis of its connection with the plexus in the anterior wall of the left ventricle as well as the carotid sinus ${ }^{[12]}$. After admission, the patient should be given adequate oxygen. Therapythey should be immediately placed in a supine position, the collar of patients should be quickly untied and the airway should be opened. At the same time, venous access should be established to do a good job in the mastery of the history as well as the dynamic changes in the progression of the disease ${ }^{[13]}$. After admission, timely give ECG monitoring, notify the relevant medical staff in the department of Cardiology at the first time, and timely treat them. Although the incidence of patients with cerebral syncope in this study did not have a high incidence of patients with neuroreflex syncope or wish syncope, its incidence in the majority of the population in China is also high.Therefore, attention should be paid in clinical practice. For patients with cerebral syncope, it is often accompanied by symptoms such as vomiting, disturbance of consciousness or complete loss of consciousness. Some scholars have speculated ${ }^{[14]}$ that it is related to the patient's neck disease or insufficient blood supply to the tissue cells of the part. For some patients with cerebral syncope, it may present with mild hemiplegia, which is generally self-limiting and difficult to heal spontaneously ${ }^{[15]}$. The clinical diagnosis and treatment of patients with this type of syncope is mainly based on conventional supine position, unraveling the collar and maintaining airway patency. On this basis, brain CT or MRI examination should be performed to clarify the condition as soon as possible in order to provide further in-depth treatment. Syncope caused by other reasons such as orthostatic hypotensive syncope is also a common disease in clinical practice.Diagnosis and treatment should be strengthened to ensure the life safety of patients. Although the common causes of syncope have been analyzed and summarized in clinical practice and the causes are various, patients are generally self-limiting. As long as the conventional supine rest and other timely treatment, syncope can obtain a good prognosis.

Syncope is a common acute condition involving multiple etiologies with distinct differences in prognosis. Emergency clinicians need to correctly judge and assess the severity of patients. For patients with severe conditions in life-threatening emergencies, emergency doctors sometimes even rely on intuition to judge the condition and intuition is based on the knowledge that has been obtained and cumulative experience. Although it has not been fully 
logical reasoning, it is an instinctive response to comprehensive thinking in a sense $\mathrm{e}^{[16]}$. The clinical thinking of emergency department and other specialties for the treatment of patients is different, and emergency medicine has a more special clinical thinking decision - "shoot first and then aim", which refers to rapid intervention for the situation endangering the life safety of patients. Then further find the cause after the vital signs are stable, and give individualized treatment in a targeted manner ${ }^{[17]}$. Corresponding symptomatic and drug treatment is taken according to different conditions. The treatment goal of syncope is to reduce recurrence and reduce mortality; the secondary goal is to prevent accidents and trauma caused by recurrence of syncope to improve the quality of life.

\section{CONCLUSION}

In summary, in order for emergency syncope patients to obtain a good therapeutic effect, emergency department doctors must assess the degree of critical condition after patient presentation.Then select appropriate therapeutic measures, determine the cause as early as possible and perform etiological treatment.In addition, different guidelines and recommendations have different backgrounds and purposes, so they should be objectively interpreted, learned and clinically applied in order to have guiding significance for the diagnosis and treatment of syncope.

\section{ACKNOWLEDGMENT}

This study was supported by the Emergency and Disaster Rescue Medical Center, The Seventh Affiliated Hospital, Sun Yat-sen University.

\section{STATEMENT}

There is no conflict of interest in this article.

\section{REFERENCES}

[1] Sheldon RS, Grubb BP, Olshansky B, et al. 2015 Heart Rhythm Society expert consensus statement on the diagnosis and treatment of postural sinus tachycardia syndrome, inappropriate tachycardia, and vasovagal syncope [J]. Heart Rhythm, 2015, 12: e41-e63.

[2] Liao Zhonghua. Clinical Decision Making in the Diagnosis and Treatment of Emergency Syncope Patients [J]. Massage \& Rehabilitation Medicine, 2021, 12 (16): 37-39.

[3] Costantino G, Sun BC, Barbic F, et al. Syncope clinical management in the emergency department: a consensus from the First International Workshop on Syncope Risk Stratification in the Emergency Department [J]. Eur Heart J, 2016, 37 (19): 1493-1498.

[4] Writing Committee Members, Shen WK, Sheldon RS, Benditt DG, et al. 2017 ACC/AHA/HRS Guidelines for the evaluation and management of patients with syncope: a report of the American College of Cardiology/American Heart Association Task Force on Clinical Practice and the Heart Rhythm Society [J]. Heart Rhythm, 2017, 14 (8): e155-e217.

[5] Chen Meifeng, Tian Haiping. Current status of research on neurally mediated syncope [J]. Medical Theory and Practice, 2021, 34 (12): 2018-2020.

[6] David G.Benditt, Gao Jie. Diagnosis and Treatment Strategy of Syncope [J]. Journal of Practical Electrocardiology, 2019, 28 (04): 238-247.

[7] Sun Qinghua, Gao Dan, Yin Lin. Etiological analysis of 131 cases of senile syncope [J]. Chinese Journal of Geriatric Cardiovascular and Cerebrovascular Diseases, 2016, 18 (11): 1178-1181. 
[8] Xu Yulong, Guan Jianguo, Zong Guirong, Liu Song. Etiology and Clinical Characteristics of 56 Cases of Syncope [J]. Chinese Journal of Clinical Health Care, 2016, 19 (05): 542-543.

[9] Tang Oushan, Zhou Haoliang, Wu Jian, Lv Jin. Follow-up analysis of 54 patients with unexplained syncope [A]. Academic Society of Electrocardiography and Pacing of Zhejiang Medical Association. Paper collection of 2016 Annual Academic Conference of Electrophysiology and Pacing of Zhejiang Medical Association [C]. Academic Branch of ECG and Pacing of Zhejiang Medical Association: Zhejiang Association of Science and Technology, 2016:1.

[10] Brignole M, Moya A, de Lange FJ, et al. ESC Scientific Document Group. 2018 ESC Guidelines for the diagnosis and management of syncope [J]. Eur Heart J, 2018, 39 (21): 1883-1948.

[11] Page RL, Joglar JA, Caldwell MA, et al. 2015 ACC/AHA/HRS Guideline for the management of adult patients with supraventricular tachycardia: a report of the American College of Cardiology/American Heart Association Task Force on Clinical Practice Guidelines and the Heart Rhythm Society [J]. J Am Coll Cardiol, 2016, 67 (13): e27-e115.

[12] Flammang D, Church TR, De Roy L, et al. ATP Multicenter Study. Treatment of unexplained syncope: a multicenter, randomized trial of cardiac pacing guided by adenosine 5 '-triphosphate testing [J]. Circulation, 2012, 125:31-36

[13] Tong Ke. Cardiac autonomic nervous function test in children with vasovagal syncope [J]. International Journal of Pediatrics, 2020, 47 (10): 677-681.

[14] Wang Guodong, Chen Liqing. Research Progress of Neutrally Mediated Syncope and Heart Rate Variability in Children [J]. Inner Mongolia Medical Journal, 2020, 52 (08): 913-916.

[15] Zhang Liyan, Wang Shuhui, Zhao Mingxia, Tu Houzhen, Zhang Yongbo. Clinical Analysis and Literature Review of Hypotension Manifested as Syncope [J]. Journal of Clinical and Experimental Medicine, 2020, 19 (14): 1563-1567.

[16] Zhu Huinan, Wang Guiyun, Lin Yanming. Emergency Treatment of Syncope in Colleges and Universities [J]. China School Doctor, 2012, 26 (02): 151 -153.

[17] Yu Jingbo, Sun Jing. Diagnosis and Treatment of Cardioinhibitory Vasovagal Syncope [J]. Journal of Zhejiang University (Medical Science), 2000 (03): 37- 40. 